\title{
SYSTEM OF OPTOELECTRONIC SENSORS FOR BREATH ANALYSIS
}

\section{Janusz Mikołajczyk ${ }^{1}$, Jacek Wojtas ${ }^{1)}$, Zbigniew Bielecki' ${ }^{1)}$, Tadeusz Stacewicz ${ }^{2}$, Dariusz Szabra'), Paweł Magryta ${ }^{2)}$, Artur Prokopiuk ${ }^{1)}$, Arkadiusz Tkacz ${ }^{1)}$, Małgorzata Panek ${ }^{1)}$}

\author{
1) Military University of Technology, Institute of Optoelectronics, Kaliskiego 2, 00-908 Warsaw, Poland \\ \janusz.mikolajczyk@wat.edu.pl,+48261839430,jacek.wojtas@wat.edu.pl,zbielecki@wat.edu.pl,dariusz.szabra@wat.edu.pl, \\ artur.prokopiuk@wat.edu.pl,arkadiusz.tkacz@wat.edu.pl,malgorzata.panek@wat.edu.pl) \\ 2) University of Warsaw, Faculty of Physics, Pasteura 5, 02-093 Warsaw, Poland \\ (Tadeusz.Stacewicz@fuw.edu.pl,Pawel.Magryta@fuw.edu.pl)
}

\begin{abstract}
The paper describes an integrated laser absorption system as a potential tool for breath analysis for clinical diagnostics, online therapy monitoring and metabolic disorder control. The sensors operate basing on cavity enhanced spectroscopy and multi-pass spectroscopy supported by wavelength modulation spectroscopy. The aspects concerning selection of operational spectral range and minimization of interference are also discussed Tests results of the constructed devices collected with reference samples of biomarkers are also presented The obtained data provide an opportunity to analyse applicability of optoelectronic sensors in medical screening.
\end{abstract}

Keywords: breath analyses, biomarkers, laser absorption spectroscopy, CEAS, MUPASS, medical screening.

C) 2016 Polish Academy of Sciences. All rights reserved

\section{Introduction}

Breath analysis is a promising field of medicine. This method offers noninvasive, point-ofcare, painless, non-stressful and real-time disease diagnostics and metabolic status monitoring. Typical human breath contains atmospheric molecules, e.g. $\mathrm{H}_{2} \mathrm{O}, \mathrm{CO}_{2}, \mathrm{O}_{2}, \mathrm{~N}_{2}$ of relatively high concentrations, several VOCs, e.g. acetone, propanol, isoprene, etc., at part per million (ppm) or sub-ppm levels, and more than 1,000 breath compounds at part per billion (ppb) or part per trillion (ppt) levels [1]. Ability of detecting these substances can provide valuable information about disorder of biochemical processes in the human body.

By now, numerous breath biomarkers have been detected using gas chromatography or mass spectroscopy $[2,3]$. But the recent advances in laser spectroscopy techniques and semiconductor lasers and detectors provide a new quality for breath analysis. The main advantages of optical detection consist in its high sensitivity and high selectivity, as equivalently offered by the MS-based techniques, but also in such features, as a near real-time response, low instrument costs, and the point-of-care (POC) function.

Other sensors (e.g. resistive gas sensors, electrochemical gas sensors) could be used as well, but they require selection of optimal detection algorithms $[4,5]$ and more enhanced constructions using new materials (e.g. nanoparticle gas sensitive films [6]).

Many breath biomarkers, such as nitric oxide, methane, carbon oxide, carbon dioxide, ammonia, acetone, ethane, and other species in the exhaled air have been detected by highsensitivity laser spectroscopic techniques [7]. Nowadays, many techniques of laser absorption spectroscopy are applied for this purpose, e.g. tunable diode laser absorption spectroscopy (TDLAS), multi-pass spectroscopy (MUPASS), wavelength modulation spectroscopy (WMS), cavity ring-down spectroscopy (CRDS), cavity enhanced absorption spectroscopy (CEAS), 
integrated cavity output spectroscopy (ICOS), cavity leak-out spectroscopy (CALOS), optical frequency comb cavity-enhanced absorption spectroscopy (OFC-CEAS), photoacoustic spectroscopy (PAS), and quartz-enhanced photoacoustic spectroscopy (QEPAS) [8]. The detection limits achieved with these techniques range from part per million (ppm) to part per trillion (ppt) levels [9]. Spectral fingerprints of the measured biomarkers are contained within the range from ultraviolet to mid-infrared [10, 11].

This paper presents an integrated laser absorption system (Sensormed Analyzer) for breath analysis developed at the Institute of Experimental Physics, Department of Physics UW and Institute of Optoelectronics MUT. For detection of methane and carbon monoxide a setup employing MUPASS and WMS was used. For measurement of nitric oxide CEAS was applied.

Carbon monoxide $(\mathrm{CO})$ and methane $\left(\mathrm{CH}_{4}\right)$ are the compounds that are contained in breath at relatively high concentrations. Their morbid level reaches $10 \mathrm{ppm}$. Such a high level provides an opportunity to perform detection with sensors working in the near-infrared range. Despite its lower sensitivity in comparison with mid-infrared wavelengths, an advantage consists in using easily available and relatively cheap diode lasers, photodetectors (photodiodes - much more sensitive than for the MIR spectral range), and standard optical devices.

Carbon monoxide is a biomarker of hyper-bilirubin, oxidative stress, respiratory infections, and asthma [12-16]. It is also used to monitor bilirubin production in smoking cessation and to access the lung diffusion capacity. For a healthy man the concentration of exhaled $\mathrm{CO}$ should not exceed $10 \mathrm{ppm}$. However, in some cases it reaches even $20 \mathrm{ppm}$ (for smokers) [17]. The best detection limit of $\mathrm{CO}$ in human breath (reaching tens and hundreds of ppb) was demonstrated in the spectral range of (4.6-5) $\mu \mathrm{m}$ using the MUPASS-WMS approach $[18,19]$. The measurement with $900 \mathrm{ppb}$ sensitivity (sufficient for medical screening) at the wavelength of $1.564 \mu \mathrm{m}$ was also reported.

Analysis of the HITRAN database [20] shows that $\mathrm{CO}$ absorption lines in the $1.56-1.57 \mu \mathrm{m}$ range are weak and strongly interfered by $\mathrm{H}_{2} \mathrm{O}$ and $\mathrm{CO}_{2}$. More efficient detection can be performed in the spectral range of around $2.33 \mu \mathrm{m}$ (Fig. 1). The absorption line of $2.33372 \mu \mathrm{m}$ is the best candidate: the $\mathrm{CO}$ absorption coefficient at $10 \mathrm{ppm}$ reaches a value of $4.4 \cdot 10^{-6} \mathrm{~cm}^{-1}$, i.e. about 50 times higher than at $1.56 \mu \mathrm{m}$. Moreover, interference by water vapour is about 200 times weaker, and by $\mathrm{CO}_{2}$ is negligible.

Methane can be detected in the NIR and MIR spectral ranges. Sensitive detection was already performed near a wavelength of $7.8 \mu \mathrm{m}$ and a range of (3.3-3.5) $\mu \mathrm{m}$ with WMS and single pass spectrophotometry. The detection limit was $0.5 \mathrm{ppm}$ which is sufficient to monitor methane in breath of a healthy human. In a near-infrared photoacoustic system $(1.63 \mu \mathrm{m})$ with laser radiation of $0.3 \mathrm{~mW}$, the limit was $6 \mathrm{ppm} \mathrm{[21].The} \mathrm{mean} \mathrm{concentration} \mathrm{of} \mathrm{methane} \mathrm{in} \mathrm{free}$ atmosphere is about $1.7 \mathrm{ppm}$, so its level in human breath also reaches higher values. Its average concentration in the exhaled air is about (3-8) ppm, but the upper limit can reach $10 \mathrm{ppm}$ for a healthy man.

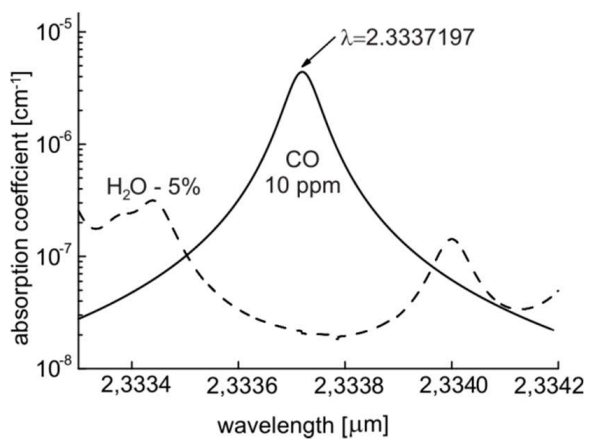

Fig. 1. The absorption spectrum of carbon oxide around the $2.3337 \mu \mathrm{m}$ line. 
Excessive concentration of $\mathrm{CH}_{4}$ has been identified as a biomarker of colonic fermentation and intestinal problems [22]. It was found that the wavelength of $2.25 \mu \mathrm{m}$ is much more profitable than that of $1.63 \mu \mathrm{m}$ (Fig. 2).

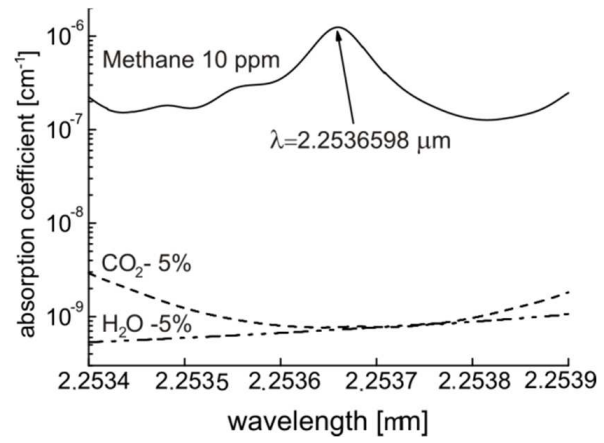

Fig. 2. The absorption spectrum of methane at the wavelength of $\sim 2.25366 \mu \mathrm{m}$.

As far as the absorption coefficients of methane for both wavelengths are similar $\left(\sim 1.2 \cdot 10^{-6}\right.$ $\mathrm{cm}^{-1}$ at concentration of $\left.10 \mathrm{ppm}\right)$ the spectral region of shorter wavelengths is strongly interfered by the carbon dioxide band with the absorption coefficient of about $0.9 \cdot 10^{-6} \mathrm{~cm}^{-1}$. Such interference is much weaker for the line of $2.25366 \mu \mathrm{m}$, since the absorption coefficients of both $\mathrm{CO}_{2}$ and $\mathrm{H}_{2} \mathrm{O}$ are over three orders of magnitude lower.

Analysis of the exhaled NO enables to monitor some diseases, e.g. asthma, hypertension, rhinitis and air way inflammation [23]. In human breath, its concentration varies from several $\mathrm{ppb}$ to several hundred $\mathrm{ppb}$. A few fingerprint absorption lines of $\mathrm{NO}$ are observed within a wavelength range of 5.260-5.266 $\mu \mathrm{m}$, reaching their maxima at $5.263 \mu \mathrm{m}$ (Fig. 3). In the air exhaled from human lungs these lines are strongly interfered by the absorption bands of water vapor and carbon dioxide. Application of a dehumidifier decreasing the $\mathrm{H}_{2} \mathrm{O}$ content by a factor of 30 is not sufficient to minimize these interferences [24]. Therefore, reduction of the sample pressure and - in this way - the decrease of broadening the collisional line is necessary. It enables to separate $\mathrm{NO}$ fingerprints from $\mathrm{CO}_{2}$ and $\mathrm{H}_{2} \mathrm{O}$ interfering lines (Fig. 3).

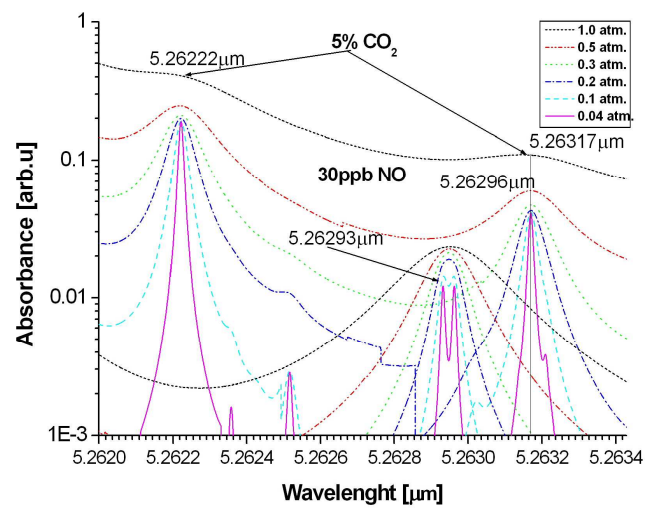

Fig. 3. The absorption spectrum of $\mathrm{NO}$ and $\mathrm{CO}_{2}$ at different pressures. The concentrations of $\mathrm{NO}(30 \mathrm{ppb})$ and $\mathrm{CO}_{2}(5 \%)$ correspond to those contained in human breath. 
As it was shown above, selected breath biomarkers are characterized by low absorption coefficients. Therefore, the sensitive techniques of measurement must be applied. These methods require laser radiation that is precisely tuned to the peak of a selected line. Thus, both linewidth and stability of the laser line should be much lower than the absorption profile width. Moreover, during selection of biomarker fingerprints the problem of minimizing interferences introduced by other compounds (especially water and carbon dioxide) must be solved. Their concentration in breath is much higher comparing with the biomarker thresholds.

\section{Integrated optoelectronic analyzing system of human breath}

The performed analysis and preliminary studies have shown that optoelectronic sensors of $\mathrm{NO}, \mathrm{CH}_{4}$ and $\mathrm{CO}$ might be an important tool in medical screening tests. Therefore, a fully automated optoelectronic breath analyzer Sensormed of the exhaled air was designed (Fig. 4).

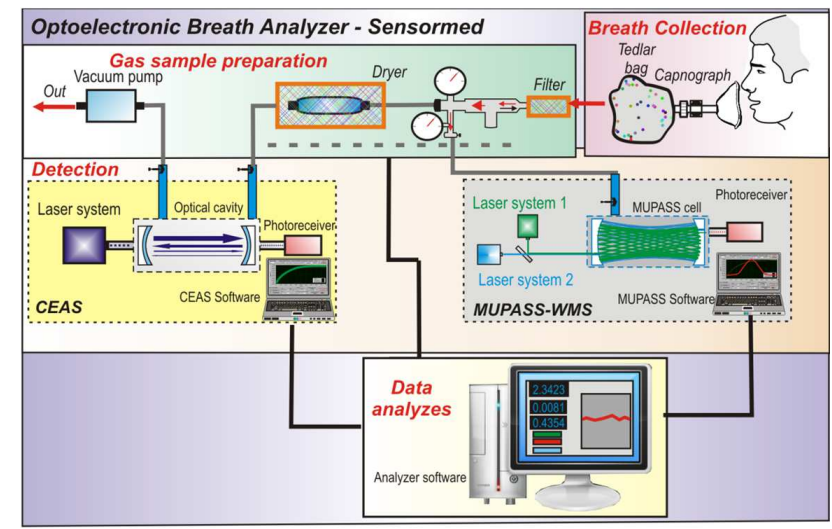

Fig. 4. A block diagram of the Sensormed Analyzer.

The analyzer consists of four main functional units: the breath collection unit, sample preparation unit, the unit of three biomarker sensors and the data analyzing unit. Each unit is equipped with its own system of signal processing and analysis, providing independent operation. The optoelectronic sensors are mounted on optical plates. The majority of signal processing circuits, as well as power and control modules, are installed in 19' rack enclosures. The main elements of the analyzer are mechanically integrated on a common platform.

The breath sampling unit is an independent device. Such configuration increases the practical usefulness of Sensormed analyzer, because it enables to prepare a sample in the off-line mode. Thanks to that the sampling unit becomes a mobile instrument for collecting the samples in Tedlar bags at any place, even outside the sensors' location. Then, the collected samples are transferred from the bag to the analyzer.

The block of data analysis is an instrument for collecting data from individual elements of the analyzer and for controlling and measuring processes.

The MUPASS-WMS sensor uses laser radiation of a wavelength that is periodically modulated within the profile of absorption line of a compound of interest. Such modulation induces the amplitude modulation of the light beam passing through the MUPASS cell containing the absorber. This approach improves selectivity and increases immunity of the detection process to noise and interferences [25-27].

For simultaneous measurements of both $\mathrm{CH}_{4}$ and $\mathrm{CO}$ concentrations two wavelength MUPASS-WMS sensors were constructed. CW single-mode DFB-diode lasers tuned to respective absorption lines of these compounds (Figs. 1 and 2) were used. The triangle- 
shaped modulation of diode currents enabled periodic scanning of laser wavelengths over the absorption lines. The observation was performed when scanning from the line peak to its right, (i.e., within ranges of $2.25366<\lambda_{1}<2.2537 \mu \mathrm{m}$ for $\mathrm{CH}_{4}$ and $2.336<\lambda_{2}<2.33372$ for $\mathrm{CO}$ ). The modulation was done with self-constructed quartz stabilized synthesizers with frequencies of $f_{1}=1 \mathrm{kHz}$ and $f_{2}=1.5 \mathrm{kHz}$ for $\mathrm{CH}_{4}$ and $\mathrm{CO}$, respectively. Using a beam splitter the radiations from both lasers were combined into a single collinear beam and directed to the MUPASS cell. Other beams spanning from the beam splitter to a monitoring photodiode enabled to control the input power of each laser.

The MUPASS cell (White design) was constructed using $0.5 \mathrm{~m}$-apart metallic mirrors with a diameter of 2 inches and a radius of curvature of $2 \mathrm{~m}$ each. The mirrors, hermetically sealed to the cell, were mounted on precise gimbals. The laser beams were introduced to the cell through a small hole ( $5 \mathrm{~mm}$ in diameter) in the reflecting layer, located at its periphery. Then, the light reflected 30 times between the mirrors and leaved the cell through a respective hole in the second mirror. The output beam, focused on photoreceiver 2, provided the signal.

Measurement of the signals from the photodiodes was carried out with a system of lock-in detectors. Two dual-input lock-in voltmeters were used. The first input worked with a reference signal of $1 \mathrm{kHz}$. It was used for measurement of input and output signals from the laser system 1, tuned to the methane spectral line. The second one operated at the frequency of $1.5 \mathrm{kHz}$ was used for signals detection from the laser system 2 , tuned to the carbon monoxide spectral line. Due to such an arrangement a significant simplification of the optical system was possible. Only one beam splitter and two photodetectors were used.

De-multiplexing the signals from both lasers was performed in an electronic way owing to the use of different modulation frequencies of both laser beams. Each lock-in provided an amplitude of the signal that was synchronous with a relevant reference trace and a relevant power of the laser beam. Due to careful selection of the working point of photodiodes and their linear output, mixing of the signals from both lasers was avoided. That enabled to eliminate cross-talk between $\mathrm{CH}_{4}$ and $\mathrm{CO}$ channels. In practice, the lock-ins were composed of a Tie-Pie $\mathrm{H} 4$ digitizer (12 bits, $50 \mathrm{MHz}$ ) with an implemented phase demodulation procedure. The demodulated signals were finally averaged over $60 \mathrm{~s}$ [28].

In the CEAS technique, the laser beam is off-axis injected into an optical cavity consisting of two high-reflection spherical mirrors. Due to multiple reflections the radiation can propagate inside the cavity. After each reflection, a small part of laser radiation leaves the cavity due to residual transmission of the mirrors. The transmitted light decreases exponentially with a defined decay time, often referred to the ring-down time. Determination of the absorption coefficient is possible by comparing the decay time values for the so-called clear cavity (reference value) and for the cavity filled with the analyzed gas. The detection limit is related to the precision of decay time measurement.

In our system, the CEAS sensor was used for detection of NO. It consists of a laser system, an optical system, a sample module and a signal processing unit. The laser system kit with a quantum sbcw1517 type laser from Alpes Lasers SA (Neuchâtel, Switzerland) with the wavelength matching the $5.2630 \mu \mathrm{m}$ NO absorption line was used. The long absorption path was ensured by the optical cavity consisting of two concave dielectric mirrors with a reflectivity of 0.995 at the wavelength of interest. An optimized PVI-2TE series detection module from VIGO System S.A. was applied to register the leakage radiation from the cavity. Its signal was digitized using an USB AD converter (C328 series from Cleverscope). The laser system employed a precise laser current driver and a temperature controller ensuring a high stability of generated wavelengths. For determining the NO concentration, a dedicated software was developed.

During the sensors tests, the gas mixtures supplied to the MUPASS cell were prepared with a system build of flow mass controllers (Beta-Erg Sp. z o. o., Poland). The reference $\mathrm{CH}_{4}$ 
concentrations were determined based on measurement of flow rates and initial concentration of gases in the gas containers. The results of testing the methane sensor are presented in Fig. 5 . There is observed a limitation of continuous control of the gas concentration. It results from the system construction providing two-stage control using two flow mass controllers. However, for both low and high concentrations, there is also observed a good linear characteristic of sensitivity. The measurement precision is better than $0.1 \mathrm{ppm}$. Because the methane concentration in breath of a healthy man should not exceed $10 \mathrm{ppm}$, this precision well fulfils the requirement for sensor monitoring of $\mathrm{CH}_{4}$ in the exhaled air.

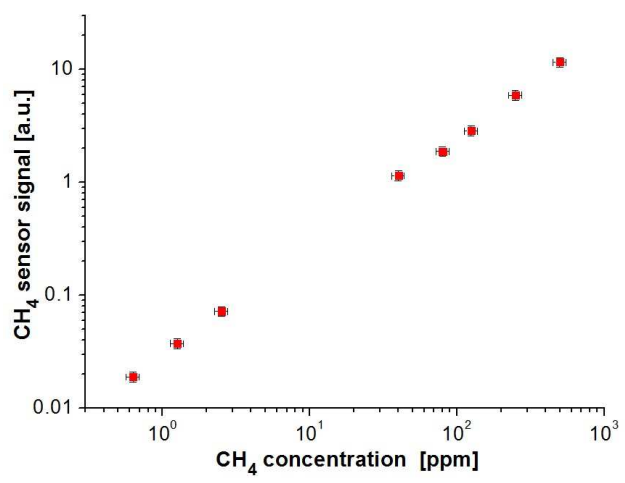

Fig. 5. The results of detection of methane with the multi-pass system.

For $\mathrm{CO}$ measurements, the reference gas concentrations were determined according to a similar procedure. Additional measurements with an MX6 iBrid Gas Detector (Industrial Scientific, USA) were performed. The results of CO detection are presented in Fig. 6. It is worth to notice a linear correlation between the results obtained using a commercially available detector and the sensor output within a concentration range of $0.4-20 \mathrm{ppm}$. It confirms applicability of the sensor for monitoring $\mathrm{CO}$ in the exhaled air for both sick and healthy man.

The NO sensor tests were performed using the reference gas mixtures at a pressure of 0.1 atm. and temperature of $296 \mathrm{~K}$. The mixtures were obtained using KIN-TEK modules: 491MB, 491M-GF and 491M-SD, equipped with a refillable type 57SA 4316 tube. The applied laser spectroscopy technique requires stable laser operation in the pulsed mode with the pulses as short as possible, therefore the pulses with the time duration of $10 \mathrm{~ns}$ and repetition frequency of $1 \mathrm{kHz}$ were used. It was also necessary to ensure a specific laser current and temperature to obtain the QC lasing wavelength matching a selected NO absorption line. An appropriate laser temperature $\left(10.3^{\circ} \mathrm{C}\right)$ and current (average $<1 \mathrm{~mA}$ at a supply voltage of $9.436 \mathrm{~V}$ ) were determined using an absorption reference cell filled with NO mixture (100 ppm). The measurement uncertainty was determined as the standard deviation of the decay time values. The results showed that the sensor provides linear detection within a range beginning from the NO concentration of $30 \mathrm{ppb}$ (Fig. 7a). Basing on measurements of the standard deviation of a blank probe (Fig. $7 \mathrm{~b})$ and commonly used three sigma $(3 \sigma)$ criterion, the limit of detection (LOD) of the nitric oxide sensor is equal to $20 \mathrm{ppb}$. It can be achieved after 300 signal averaging procedures that requires only 2 seconds.

According to the ATS recommendation (the morbid level of $50 \mathrm{ppb}$ and $35 \mathrm{ppb}$ for adults and children, respectively), the sensor can be applied in detection of NO gas as a symptom of atopic asthma, eosinophilic bronchitis or COPD with mixed inflammatory phenotype [29, 30]. 


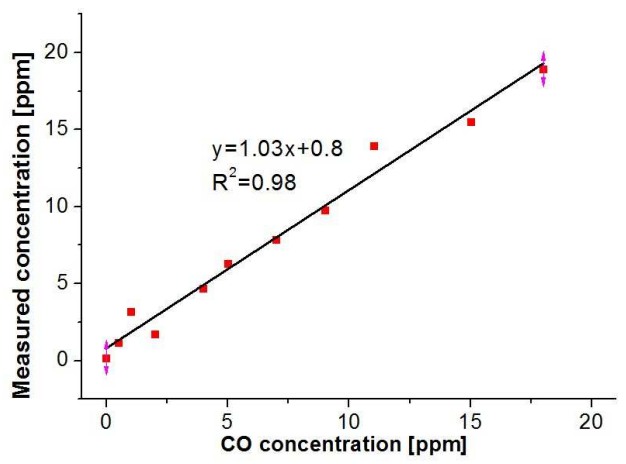

Fig. 6. The results of $\mathrm{CO}$ sensor test.

a)

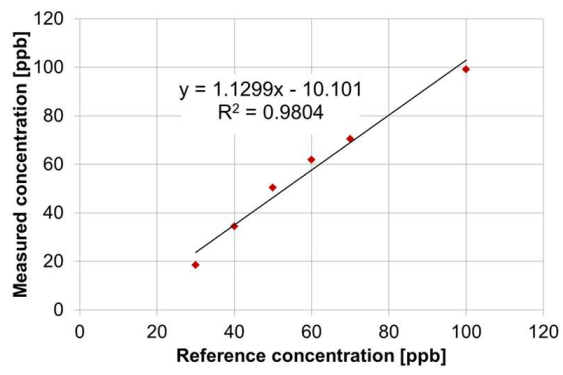

b)

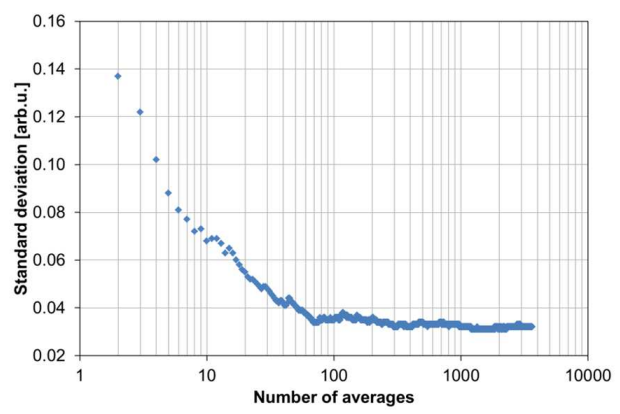

Fig. 7. a) An example of the NO sensor test results: the calibration characteristics; b) the standard deviation with the averaging procedure.

\section{Conclusion}

The work presents some practical aspects concerning detection of biomarkers with the use of optoelectronic methods. There are described the sensors constructed for detection of NO, $\mathrm{CH}_{4}$ and $\mathrm{CO}$. Their operation concept is based on cavity enhanced (CEAS) and wavelength modulation spectroscopy (WMS). The results of experiments showed that the sensors are sensitive enough to analyze human breath.

For the methane sensor, the detection limit of $\sim 0.1 \mathrm{ppm}$ was obtained. This value is significantly lower than the upper limit of methane concentration in breath of a healthy man $(10 \mathrm{ppm})$. Therefore, this sensor satisfies the requirements for screening diseases and therapy monitoring. The carbon oxide sensor is characterized by a linear response in the concentration range higher than $0.4 \mathrm{ppm}$. Comparing it with the concentration limit for a healthy man $(10 \mathrm{ppm})$, this system is well suited for determination of disease states (e.g. liver diseases).

For the nitrogen oxide sensor, the obtained detection limit was $30 \mathrm{ppb}$. According to the ATS recommendation such a limit of NO detection is sufficient to monitor people's health state and to detect respiratory diseases, like asthma or chronic bronchitis.

The presented optoelectronic system for human breath analysis can be used as a screening tool in medicine doctors' cabinets, clinics and consulting-rooms. Elaboration and implementation to the clinical practice of an early-warning desktop system for real-time disease detection are also important. Such a device should be simple and cheap. Simultaneously, its sensitivity should meet the requirements for medical diagnostics. 


\section{Acknowledgements}

The research presented in this paper has been supported by the National Centre for Research and Development in the scope of Project No.: 179900 (PBS1/A3/7/2012).

\section{References}

[1] Wang, Ch., Sahay, P. (2009). Breath analysis using laser spectroscopic techniques: breath biomarkers, spectra fingerprints, detection limit. Sensors, 9, 8230-8262.

[2] Buszewski, B., Grzywinski, T., Ligor, T., Stacewicz, T., Bielecki, Z., Wojtas, J. (2013). Detection of volatile organic compounds as biomarkers in breath analysis by different analytical techniques. Bioanalysis, 5(18), $2287-306$.

[3] Amann, A., Ligor, M., Ligor, T., Bajtarevic, A., Ager, C., Pienz, M., Denz, H., Fiegl, M., Hilbe, W., Weiss, W., Lukas, P., Jamnig, H., Haidenberger, A., Sponring, A., Filipiak, W., Miekisch, W., Schubert, J., Troppmair, J., Buszewski, B. (2010). Analysis of exhaled breath for screening of lung cancer patients. Magazine of European Medical Oncology, 3, 106-112.

[4] Lentka, Ł., Smulko, J.M., Ionescu, R., Granqvist, C.G., Kish, L.B. (2015). Determination of Gas Mixture Components Using Fluctuation Enhanced Sensing And The LS-SVM Regression Algorithm. Metrol. Meas. Syst., 22(3), 341-350.

[5] Kalinowski, P., Woźniak, Ł., Strzelczyk, A., Jasinski, P., Jasinski, G. (2013). Efficiency of linear and nonlinear classifiers for gas identification from electrocatalytic gas sensor. Metrol. Meas. Syst., 20(3), 501-512.

[6] Heszler, P., Ionescu, R., Llobet, E., Reyes, L.F., Smulko, J.M., Kish, L.B., Granqvist, C.G. (2007). On the selectivity of nanostructured semiconductor gas sensors. Physica status solidi (b), 244(11), 4331-4335.

[7] McCurdy, M.R., Bakhirkin, Y., Wysocki, G., Lewicki, R., Tittel, F.K. (2007). Recent advances of laserspectroscopy based techniques for applications in breath analysis. J. Breath Res., 1, R1-R12.

[8] Shorter, J.H., Nelson, D.D., McManus, J.B., Zahniser, M.S., Sama, S.R., Milton, D.K. (2011). Clinical study of multiple breath biomarkers of asthma and $\mathrm{COPD}\left(\mathrm{NO}, \mathrm{CO}_{2}, \mathrm{CO}\right.$ and $\left.\mathrm{N}_{2} \mathrm{O}\right)$ by infrared laser spectroscopy. J. Breath Res., 5, DOI: 10.1088/1752-7155/5/3/037108, 1-12.

[9] Shorter, J.H., Nelson, D.D., McManus, J.B., Zahniser, M.S., Milton, D.K. (2010). Multicomponent breath analysis with infrared absorption using room-temperature quantum cascade lasers. IEEE Sensors Journal, $10,76-82$.

[10] Wysocki, G., McCurdy, M.R., So, S., Rolller, C., Tittel, F.K. (2005). Conference: Breath Analysis for Clinical Diagnosis and Therapeutic Monitoring. DOI: 10.1142/9789812701954_006.

[11] Nwaboh, J.A., Persijn, S., Heinrich, K., Sowa, M., Hering, P., Werhahn, O. (2012). QCLAS and CRDSbased CO uanification as aimed at in breath measurements. Int. Journal of Spectroscopy, DOI: 10.1155/2012/894841, 1-10.

[12] Vreman, H.J., Mahoney, J.J., Stevenson, D.K. (1995). Carbon monoxide and carboxyhemoglobin. Adv. Pediatr., 42, 330-334.

[13] Stevenson, D.K., Vreman, H.J. (1997). Carbon monoxide and bilirubin production in neonates. Pediatr. Rev., $100,252-259$.

[14] Applegate, L.A., Luscher, P., Tyrrell, R.M. (1991). Induction of heme oxygenase: a general response to oxidant stress in cultured mammalian cells. Cancer Res., 51, 974-978.

[15] Yamaya, M., Sekizawa, K., Ishizuka, S., Monma, M., Mizuta, K., Sasaki, H. (1998). Increased carbon monoxide in exhaled air of subjects with upper respiratory tract infections. Am. J. Respir. Crit. Care Med., $158,311-314$.

[16] Zayasu, K., Sekizawa, K., Okinaga, S., Yamaya, M., Ohrui, T., Sasaki, H. (1997) Increased carbon monoxide in exhaled air of asthmatic patients. Am. J. Respir. Crit. Care Med., 156, 1140-1143.

[17] Thorpe, M.J., Moll, K.D., Jones, J.R., Safdi, B., Ye, J. (2006). Broadband cavity ringdown spectroscopy for sensitive and rapid molecular detection. Science, 311, 1595-1599. 
[18] Moeskops, B.W., Cristescu, S.M., Harren, F.J. (2006). Sub-part-per-billion monitoring of nitric oxide by use of wavelength modulation spectroscopy in combination with a thermoelectrically cooled, continuous-wave quantum cascade laser. Opt. Lett., 31, 823-825.

[19] Moskalenko, K.L., Nadezhdinskii, A.I., Adamovskaya, I.A. (1996). Human breath trace gas content study by tunable diode laser spectroscopy technique. Infrared Phys. Tech., 37, 181-192.

[20] Rothman, L.S., Gordon, I.E., et al. (2013). The HITRAN 2012 Molecular Spectroscopic Database. J. Quant Spectrosc Radiation transfer, 130, 4-50.

[21] Scotoni, M., Rossi, A., Bassi, D., Buffa, R., Iannotta, S., Boschetti, A. (2006). Simultaneous detection of ammonia, methane and ethylene at $1.63 \mu \mathrm{m}$ with diode laser photoacoustic spectroscopy. Appl. Phys. B: Lasers Opt., 82, 495-500.

[22] Le Marchand, L., Wilkens, L.R., Harwood, P., Cooney, R.V. (1992). Use of breath hydrogen and methane as markers of colonic fermentation in epidemiologic studies: circadian patterns of excretion. Environ. Health Perspect., 98, 199-202.

[23] Birrell, M.A., McCluskie, K., Hardaker, E., Knowles, R., Belvisi, M.G. (2006). Utility of exhaled nitric oxide as a noninvasive biomarker of lung inflammation in a disease model. Eur. Respir. J., 28, 1236-1244.

[24] Wojtas, J. (2015). Application of Cavity Enhanced Absorption Spectroscopy to the Detection of Nitric Oxide, Carbonyl Sulphide, and Ethane-Breath Biomarkers of Serious Diseases. Sensors, 15, 14356-14369.

[25] Bomse, D.S., Stanton, A.C., Silver, J.A. (1992). Frequency modulation and wavelength modulation spectroscopies: comparison of experimental methods using a lead salt diode laser. Applied Optics, 31, 718-731, DOI: 10.1364/AO.31.000718.

[26] Oh, D.B., Paige, M.E., Bromse, D.S. (1998). Frequency modulation multiplexing for simultaneous detection of multiple gases by use of wavelength modulation spectroscopy with diode lasers. Appl. Optics, 37 , 2499-2501.

[27] Rieker, G.B. (2009). Wavelength-modulation spectroscopy for measurements of gas temperature and concentration in harsh environmnents. A dissertation submitted to the Department of Mechanical Engineering and The Committee on Graduate Studies of Stanford University.

[28] Patent application P.416703 entitled "The set-up and the method for the detection of gases based on multiplexing and demultiplexing of optical signals in laser spectroscopy" invented by T. Stacewicz, P. Magryta, Z. Bielecki and J. Wojtas has been submitted to the Polish Patent Office on 31.03.2016.

[29] http://www.thoracic.org/about/overview.php (Mar. 2015).

[30] http://www.ersnet.org/images/stories/pdf/ERS_Annual_report_1314.pdf(March 2015). 